\title{
End Point of the Ultraspinning Instability and Violation of Cosmic Censorship
}

\author{
Pau Figueras, ${ }^{1, *}$ Markus Kunesch, ${ }^{2, \dagger}$ Luis Lehner, ${ }^{3, \ddagger}$ and Saran Tunyasuvunakool ${ }^{2, \$}$ \\ ${ }^{1}$ School of Mathematical Sciences, Queen Mary University of London, Mile End Road, London E1 4NS, United Kingdom \\ ${ }^{2}$ Department of Applied Mathematics and Theoretical Physics (DAMTP), Centre for Mathematical Sciences, University of Cambridge, \\ Wilberforce Road, Cambridge CB3 OWA, United Kingdom \\ ${ }^{3}$ Perimeter Institute for Theoretical Physics, Waterloo, Ontario N2L 2Y5, Canada
}

(Received 22 February 2017; published 11 April 2017)

\begin{abstract}
We determine the end point of the axisymmetric ultraspinning instability of asymptotically flat Myers-Perry black holes in $D=6$ spacetime dimensions. In the nonlinear regime, this instability gives rise to a sequence of concentric rings connected by segments of black membrane on the rotation plane. The latter become thinner over time, resulting in the formation of a naked singularity in finite asymptotic time and hence a violation of the weak cosmic censorship conjecture in asymptotically flat higherdimensional spaces.
\end{abstract}

DOI: 10.1103/PhysRevLett.118.151103

Introduction.-The recent detection of gravitational waves from black hole binary mergers $[1,2]$ has provided the first direct observation of these objects. The current observational data are compatible with the predictions of general relativity, and they suggest that the end point of such mergers is a Kerr black hole (BH) [3]. These observations provide evidence that the Kerr $\mathrm{BH}$ in vacuum is nonlinearly stable, at least within a certain range of the angular momentum. However, a mathematically rigorous understanding of the stability of the generic Kerr BH, as well as a thorough understanding of its dynamics under arbitrary perturbations, is still lacking. In fact, recent work suggests that novel and nontrivial dynamics may be present very close to extremality (e.g., Refs. [4-6]).

Higher dimensional BHs, however, can be unstable under gravitational perturbations. This was first shown by Gregory and Laflamme (GL) for black strings and black $p$-branes [7]. Determining the end point of this instability has been a subject of intense study due to the potential implications on the weak cosmic censorship conjecture (WCC) in such spacetimes. With the aid of numerical relativity (NR), Ref. [8] found that the GL instability gives rise to a self-similar structure of bulges connected by ever thinner string segments, which all undergo the GL instability. Eventually, the black string pinches off in finite asymptotic time, resulting in a naked singularity. Since no fine-tuning of the initial data was required, this result constituted a violation of the WCC, albeit in spacetimes with compact extra dimensions.

Contrary to the $D=4$ case, asymptotically flat $\mathrm{BHs}$ in higher dimensions can carry arbitrarily large angular momenta. At very large angular momenta, BHs become highly deformed and resemble black branes, which are known to be unstable under the GL instability [9]. This observation highlighted the possibility that higher dimensional asymptotically flat $\mathrm{BHs}$ can be unstable under gravitational perturbations. This indeed turned out to be the case. For instance, the black rings of Ref. [10] suffer from various types of instabilities [11-16], including the GL instability. The nonlinear evolution of the latter was studied in a very recent work by three of us [15], where it was found that, for sufficiently thin rings, the evolution of the instability is similar to that of the GL instability of black strings. Hence, a naked singularity should form in finite asymptotic time, thus violating the WCC in higherdimensional asymptotically flat spaces. However, the calculations in Ref. [15] were computationally highly demanding, which limited the extent to which the instability could be explored. It was therefore not possible to estimate the time scale of a possible pinch-off or to determine whether the process is self-similar as for black strings.

Reference [9] conjectured (and Ref. [17] later confirmed) that rapidly spinning Myers-Perry (MP) BHs $[18,19]$ in $D \geq 6$ are unstable under a GL-type of instability, which is referred to as the "ultraspinning instability." As with the GL case, there exist zero modes that connect MP BHs with different families of "bumpy" BHs [20,21]. In this Letter, we report on the final stages of the evolution of the ultraspinning instability of singly spinning MP BHs in 6 dimensions. We restrict ourselves to the instability that deforms the horizon without breaking any of the rotational symmetries of the background, i.e., the axisymmetric one. The imposed symmetries reduce the problem to a system of $(2+1)$-dimensional partial differential equations (PDEs), which is significantly more computationally tractable than the one described in Ref. [15]. This allows us to elucidate the dynamics of the ultraspinning instability in full detail.

Numerical methods. -We solve the $D=6$ vacuum Einstein equation numerically with a $U(1) \times S O(3)$ isometry imposed. The ultraspinning instability lies within this symmetry sector, but other nonaxisymmetric instabilities which are not captured by this ansatz do exist. We impose the symmetry using the modified cartoon method [22-24]. We employ the CCZ4 formulation $[25,26]$ on a Cartesian 

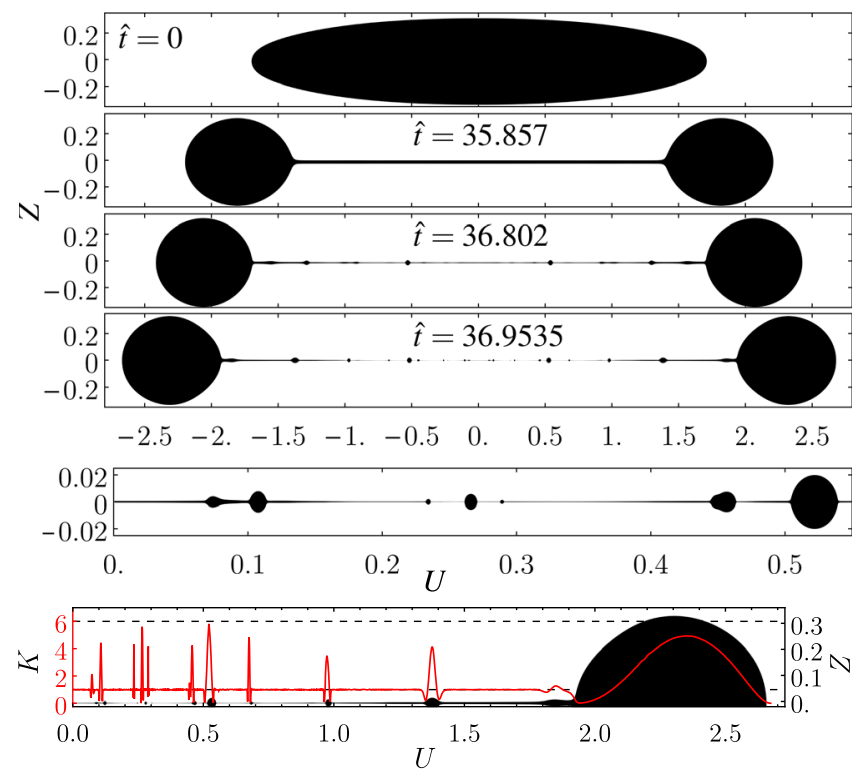

FIG. 1. Top: Embedding diagrams of the AH at different stages of the evolution of the ultraspinning instability of a MP BH with $a / \mu^{\frac{1}{3}}=1.7$. Here $\hat{t}=t / \mu^{\frac{1}{3}}$. The structure of rings that form on the membrane in the middle depends sensitively on the grid setting and is not convergent [28]. Bottom: Normalized spacetime Kretschmann invariant pulled back onto the $\mathrm{AH}$.

grid with the redefinition of the constraint damping parameter $\kappa_{1} \rightarrow \kappa_{1} / \alpha$, where $\alpha$ is the lapse function [27]. Typically, we choose $\kappa_{1}=0.5$ and $\kappa_{2}=0$. As initial data, we take the 6-dimensional singly spinning MP BH,

$$
\begin{aligned}
d s^{2}= & -d t^{2}+\frac{\mu}{r \Sigma}\left(d t-a \sin ^{2} \theta d \phi\right)^{2}+\frac{\Sigma}{\Delta} d r^{2}+\Sigma d \theta^{2} \\
& +\left(r^{2}+a^{2}\right) \sin ^{2} \theta d \phi^{2}+r^{2} \cos ^{2} \theta d \Omega_{(2)}^{2},
\end{aligned}
$$

with a new quasiradial coordinate $\rho$ defined by

$$
r=\rho\left(1+\frac{1}{4} \frac{r_{h}^{3}}{\rho^{3}}\right)^{2 / 3},
$$

where $\mu$ and $a$ are the mass and rotation parameters, respectively, $\Sigma=r^{2}+a^{2} \cos ^{2} \theta, \Delta=r^{2}+a^{2}-\mu / r$, and $r_{h}$ is the largest real root of $\Delta\left(r_{h}\right)=0$. In our simulations, we set $\mu=1$ and consider MP BHs with $1.5 \leq a / \mu^{\frac{1}{3}} \leq 2.0$. The first (ring-shaped) unstable mode sets in at $a / \mu^{\frac{1}{3}}=$ 1.572 and the second (saturn-shaped) mode sets in at $a / \mu^{\frac{1}{3}}=1.849$ [17].

We evolve the lapse and the shift using the CCZ4 $(1+\log )$ slicing with an advection term and the variant of the GammaDriver shift condition used in Ref. [15] (see also the Supplemental Material [28]). Initially, we choose $\alpha=\chi$ and $\beta^{i}=\chi \beta_{\mathrm{MP}}^{i}$, where $\beta_{\mathrm{MP}}^{i}$ is the analytic shift obtained from Eq. (1) and $\chi$ denotes the conformal factor. To help stabilize the evolution, we add diffusion terms well inside the apparent horizon (AH) as described in Ref. [15]. The coordinate singularity present in our initial data is regularized by the "turduckening" method [32,33]. Since the gauge in Eq. (1) is not optimal, we first evolve this initial data until the gauge has settled to spatial harmonic coordinates with respect to the conformal metric. In this new gauge, the shape of the AH flattens and resembles a pancake for rapidly spinning BHs (see Fig. 1), as one would expect on physical grounds [9]. We stress that this gauge adjustment process occurs over a short time period, during which we have verified that there is no significant physical evolution.

Once the gauge dynamics has settled, we trigger the ultraspinning instability by perturbing the conformal factor via

$\chi=\chi_{0}\left\{1+A J_{0}\left[j_{0, k} \sin \left(\frac{\pi}{2} \sigma\right)\right] \exp \left[-\left(\frac{\chi_{0}}{\chi_{h}}-\frac{\chi_{h}}{\chi_{0}}\right)^{2}\right]\right\}$,

where $A$ is the amplitude of the perturbation, $\chi_{0}$ is the unperturbed conformal factor, $\chi_{h}$ is the value of the unperturbed conformal factor at the horizon, $J_{0}$ is the Bessel function of the first kind, $j_{0, k}$ is the $k$ th zero of $J_{0}$, and $\sigma=\sqrt{x^{2}+y^{2}} / \tilde{R}$. Here $\tilde{R}$ is a parameter that determines the extent of the deformation in the rotation plane, and $x$ and $y$ are our Cartesian coordinates. The expression Eq. (3) ensures that the perturbation is localized on the horizon and behaves like $J_{0}$ near the rotation axis, where $J_{0}$ captures the unstable mode reasonably accurately [9]. This perturbation introduces constraint violations, but they are small and depend linearly on the amplitude of the perturbation. In our simulations, we check that these constraint violations decay exponentially with time (thanks to the CCZ4 constraint damping terms), and that the physical parameters of the perturbed $\mathrm{BH}$ change by less than $1 \%$ compared to those of the unperturbed $\mathrm{BH}$.

To understand the end point of the ultraspinning instability, we monitor the geometry of the AH. Most traditional approaches in NR assume that the AH can be given by the level set of a function of the angular coordinates (see Ref. [34] for a review). In our current symmetry setting this would mean $r=R(\theta)$, where $r$ is the radial coordinate and $\theta$ is the polar angle on the sphere. However, in the final stages of the ultraspinning instability, this is not a valid assumption as $R(\theta)$ fails to be a single-valued function (see Fig. 1). To overcome this problem, we consider the $\mathrm{AH}$ as a completely general parametric surface $(x(u), y(u), z(u))$, where $u$ is the parameter. We then solve the elliptic PDEs that arise from setting the expansion and a gauge condition for $u$ to zero. The technical details of this construction can be found in the Supplemental Material [28] and in Ref. [35].

We solve the CCZ4 equations numerically with the GRChombo code $[36,37]$ using up to 22 levels of refinement (each refined in a 2:1 ratio) with a coarsest grid spacing of $0.35 \mu^{\frac{1}{3}}$. We discretize the equations using fourth-order finite differences and integrate in time using RK4. We choose our refinement levels such that the $\mathrm{AH}$ is covered by at least 
TABLE I. Growth rates of the first unstable mode. Errors are $\pm 3 \%$ for $a / \mu^{\frac{1}{3}} \geq 1.7$ and $\pm 25 \%$ for $a / \mu^{\frac{1}{3}}=1.6$.

\begin{tabular}{llllll}
\hline \hline$a / \mu^{\frac{1}{3}}$ & 1.6 & 1.7 & 1.8 & 1.9 & 2.0 \\
$\Im \varpi \mu^{\frac{1}{3}}$ & 0.020 & 0.130 & 0.213 & 0.262 & 0.299 \\
\hline \hline
\end{tabular}

57 points at all times. Convergence studies indicate that the order of convergence is $\approx 3$. Some relevant numerical tests are presented in the Supplemental Material [28].

Results.-In Fig. 1 (top) we present different snapshots of the embedding of a constant rotational angle section of the $\mathrm{AH}$ into $\mathbb{R}^{4}$ at representative stages of the evolution [38]. In the range of $a / \mu^{\frac{1}{3}}$ that we have explored, the ring mode grows fastest and governs the nonlinear evolution. We find that initially only a large ring forms at the outermost edge of the horizon [second snapshot in Fig. 1 (top)], even if we perturb with a "saturn-shaped" perturbation by setting $k=2$ in (3). In Table I, we summarize the growth rates of the first unstable mode for different values of $a / \mu^{\frac{1}{3}}$ as calculated from our simulations. To our knowledge, these are not currently available in the literature.

To estimate how much mass and angular momentum are contained within the outermost ring, we calculate the corresponding Komar integrals on the AH. The calculated mass is only accurate once the system has settled down to a steady state. Towards the end of our simulations, the Komar mass changes by less than $1 \%$, thus indicating that the majority of the AH has settled down sufficiently. We find that the outermost ring accounts for $98 \%-99 \%$ of the total mass and more than $99.99 \%$ of the angular momentum. The radiated mass is too small $(<2 \%)$ to be distinguishable from changes in the Komar mass due to the system not having settled down completely. Angular momentum is conserved because of our symmetry assumptions.

After the outermost large ring has formed, the region of the horizon connecting it to the rotation axis resembles a thin, locally boosted black membrane (see Fig. 1). The evolution of the ultraspinning instability takes place in the radial direction, while the local boost is along an orthogonal $U(1)$ direction. Therefore, the dynamics of the black membrane under this instability should be insensitive to the local boost and, since the transverse direction is flat, similar to the GL dynamics of a $5 \mathrm{D}$ black string. The portion of the $\mathrm{AH}$ that resembles a black membrane is indeed GL unstable and can accommodate many unstable modes (see Table II). Its subsequent evolution leads to a sequence of ever thinner rings connected by segments of black membrane which are GL unstable.

As evidence that the horizon has the geometry of concentric rings connected by membrane sections, we evaluate the (suitably normalized) spacetime Kretschmann invariant, $K=R_{a b c d} R^{a b c d} Z_{\mathrm{AH}}^{4} / 12$, on the $\mathrm{AH}$. Here $Z_{\mathrm{AH}}$ is the radius of the transverse sphere, which measures the thickness of the $\mathrm{AH}$. The normalization is such that $K=1$ for a black membrane and $K=6$ for a $6 \mathrm{D}$ black string. The results [Fig. 1(bottom)] are in close agreement with $K=1$ on the membrane sections and approach $K=6$ on most of the fully formed rings, suggesting that they are well approximated by stationary black strings.

There are three fundamental differences between the dynamics of unstable black strings and ultraspinning MP BHs. First, the latter have compact horizons that do not wrap any topological direction in spacetime. Thus, any selfsimilarity is broken near the edges, and also in the early stages of the instability when the radial extent of the unstable membrane sections is comparable to the size of the whole BH. Second, MP BHs are rotating and the imposition of axisymmetry introduces a new constraint: the conservation of angular momentum. Furthermore, the rotation causes a centrifugal force which redistributes angular momentum outwards and leads to different membrane sections having different thicknesses (see third snapshot in Fig. 1). Hence, the local GL instabilities of each membrane section evolve on different time scales. Third, throughout the whole evolution, the small concentric rings that form after the first generation move around, causing significant additional stretching over the time it takes to form a new generation. The nonzero boost velocity imparted upon the membrane delays the formation of the $i$ th [39] generation, while the stretching itself also causes the membrane to become thinner, resulting in the earlier formation of the $(i+1)$ th generation.

The combination of these three effects implies that the evolution of the ultraspinning instability is not self-similar: while we do observe newly formed membrane sections all undergoing the GL instability, the time elapsed between the formation of successive generations does not decrease with a universal factor (c.f. Table II), even for later generations. Instead, in the $a / \mu^{\frac{1}{3}}=1.7$ run, we observe factors between 0.07 and 0.42 . Furthermore, they cause the pinch-off to happen sooner, mostly due to the quick drop in the formation times between generations at the beginning. The largest factor between generations that we observed was $X_{\max }=0.41$. Since $X_{\max }<1$, we can bound the pinchoff time by a geometric series

$t_{c}<t_{0}+\left(t_{1}-t_{0}\right) \sum_{i} X^{i}<t_{0}+\left(t_{1}-t_{0}\right) /\left(1-X_{\max }\right)$.

While this upper bound is not sharp, it provides evidence that the $\mathrm{BH}$ pinches off in finite asymptotic time.

From Table II, we see that the typical ratio $R_{i} / L_{i}$ between the thickness and the length of a membrane section varies between 300 and 600 . For the GL instability of black strings, this ratio is approximately 100 across generations [8]. Therefore, the membranes that form in the evolution of the ultraspinning instability are more unstable, indicating a faster pinch-off time.

Let us now explain the local dynamics which leads to the nonconstant factors between generations. We calculate the radial velocity $d r / d t$ of null rays which corotate with the $\mathrm{BH}$ to estimate the local radial flow velocity of the $\mathrm{AH}$. The results are shown in Fig. 2. They paint a very consistent 
TABLE II. Properties of the generations. The ratio of length to thickness of the $i$ th generation membrane was measured just before the formation of the $(i+1)$ th generation. The time it takes to form the next generation decreases with factors $0.07,0.41$, and 0.26 .

\begin{tabular}{llllll}
\hline \hline Generation & 1 & \multicolumn{1}{c}{2} & \multicolumn{1}{c}{3} & \multicolumn{1}{c}{4} & \multicolumn{1}{c}{5} \\
\hline$t_{i} / \mu^{\frac{1}{3}}$ & 31.8 & 36.45 & 36.78 & 36.916 & 36.952 \\
$L_{i} / Z_{\mathrm{AH}, i}$ & 540 & 530 & 370 & 510 & $>370$ \\
\hline \hline
\end{tabular}

picture: near each ring, the radial velocity either decreases or reverses completely, leading to a build up of mass. This also explains the numerous sign changes around the thinnest point of the membrane, where many higher generation rings are present.

The outermost ring very quickly settles down to an almost stationary state. However, as Fig. 2 (top) shows, it is still rigidly expanding outwards in the rotation plane. Compared with the balanced 6D black rings [20,40], we find that the area and angular velocity are still $7 \%$ below and $15 \%$ above their respective equilibrium values in the final frame of our simulation. These values are consistent with the fact that the ring still has to expand by an additional $7 \%$ in the rotation plane in order to reach the equilibrium $S^{1}$ radius while conserving angular momentum.
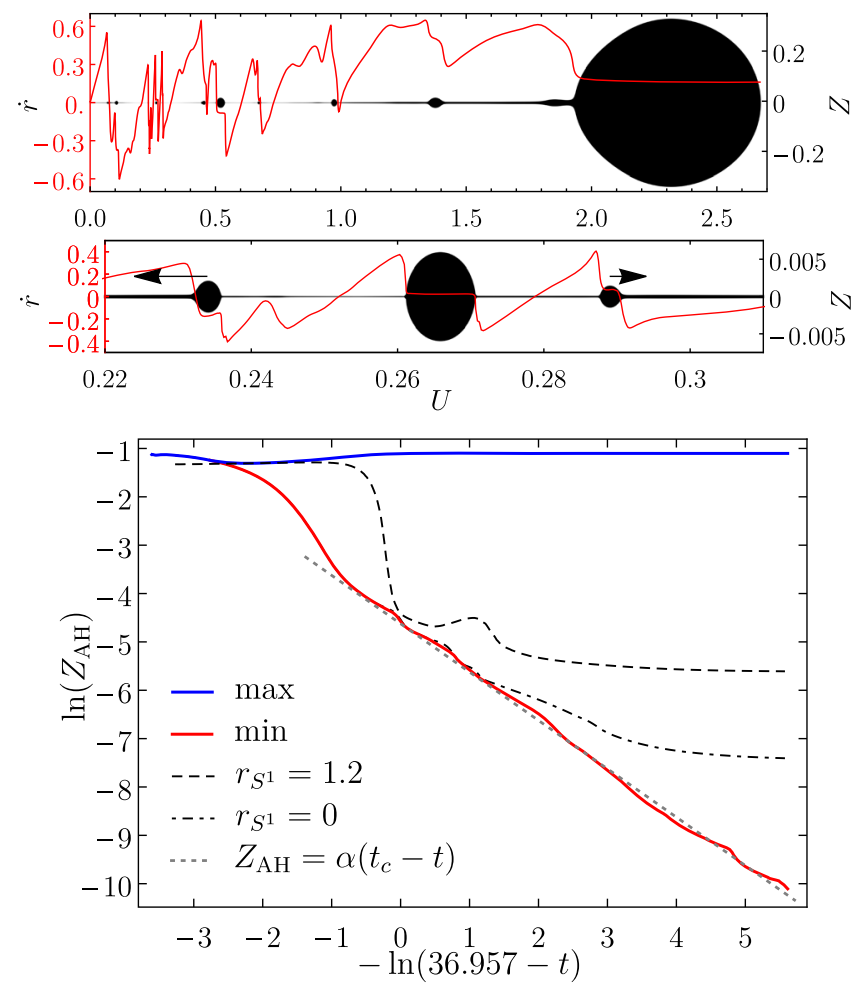

FIG. 2. Top: Radial velocity $\dot{r} \equiv d r / d t$ of a null ray moving with the AH. Middle: Zoom of the thinnest region. The arrows indicate the direction of the local velocity of the rings. Bottom: Evolution of the $\mathrm{AH}$ thickness at several representative locations for $a / \mu^{\frac{1}{3}}=1.7$.
At late times, Fig. 2 shows that the flow in the $U<1$ region is unaffected by the pull from the outermost ring, and the dynamics is therefore determined by the higher generation rings. These differ from the first generation in that they carry far too little angular momentum to be balanced. Instead, they are held in place by the tension of the membrane sections surrounding them. The tension of a membrane in $6 \mathrm{D}$ is proportional to its thickness, and different parts of the membrane have different thickness due to the pull from the outermost ring from the outset. These differences are amplified as the GL instability develops on each of these local sections. As the thicknesses of the surrounding membranes change, higher generation rings develop a radial velocity towards the thicker sections. This is clearly visible in Fig. 2 (middle), and is large enough to significantly change the width of a membrane section during the development of a new generation, thus affecting its formation time.

To obtain a precise value for the pinch-off time, we track the global minimum thickness of the membrane. Even though the dynamics of higher generation rings prevents the formation of new generations from being self-similar, the minimum thickness closely follows the scaling law

$$
Z_{\mathrm{AH}}=\alpha\left(t_{c}-t\right),
$$

similar to black strings $[8,41]$ and the Rayleigh-Plateau instability of fluid columns [see Fig. 2 (bottom)]. This strongly supports our earlier conclusion that the $\mathrm{BH}$ will pinch off in finite asymptotic time, $t_{c}$, giving rise to a naked singularity. By performing a 2-parameter fit with Eq. (5), we can obtain values for the pinch-off time $t_{c}$ and the dimensionless constant $\alpha$. The value for the latter, $\alpha=(9.9 \pm 0.2) \times 10^{-3}$, is universal in that it is the same for all of our runs and is independent of the rotation parameter and initial data.

We may finally speculate about the end point. Figure 2 suggests that after pinch-off the outermost ring will settle down to its balanced configuration, absorbing the nearby $(U \gtrsim 1)$ membrane section. Since the angular velocity in the central sections of the membrane is much too low to form balanced rings, sections closer to the center will collapse into a spherical $\mathrm{BH}$ with negligible angular momentum. Therefore, the end point will be a black saturn in 6D. However, it will not be the saturn that maximizes the entropy for a given final mass and angular momentum, which consists of a central BH carrying all the mass surrounded by a thin ring that accounts for all the angular momentum [42]. Nevertheless, we find that as $a / \mu^{\frac{1}{3}}$ is increased, the end point becomes more similar to this optimal configuration.

Discussion. - Our results provide evidence that the ultraspinning instability evolves into a naked singularity in finite asymptotic time, and thus can be interpreted as a potential counterexample to the WCC in higher-dimensional asymptotically flat spaces. In the approach to pinch-off, the minimum membrane thickness very closely follows a scaling 
law, Eq. (5), with a universal constant $\alpha$. However, in $D \geq 6$ MP BHs are unstable to nonaxisymmetric modes [23,43,44]. Therefore, to find a generic violation of the WCC in MP BH spacetimes, one has to consider the evolution under all these instabilities. Work in this direction is underway. Since the growth rate of the bar mode instability saturates [44], we expect that for sufficiently rapidly spinning MP BHs the ultraspinning instability will dominate. Once the first generation ring has formed, the membrane inside becomes thinner by a factor of 50 . Thus, the ultraspinning instability in this Letter is an order of magnitude faster than the axisymmetry-breaking GL instability of the outermost ring. Therefore, we expect that for sufficiently large values of $a / \mu^{\frac{1}{3}}$, modes that preserve the axisymmetry are dominant in all stages of the evolution, and that the violation of the WCC presented in this Letter should be generic.

We thank Roberto Emparan for discussions and Juha Jäykkä and Kacper Kornet for their technical support. We thank the GRChombo team for the great collaboration. Part of the computations for this Letter were undertaken on the COSMOS Shared Memory system at DAMTP (University of Cambridge). COSMOS is operated on behalf of the STFC DiRAC HPC Facility and is funded by BIS National Einfrastructure capital Grant No. ST/ J005673/1 and STFC Grants No. ST/H008586/1, No. ST/ K00333X/1. We thankfully acknowledge the computer resources at MareNostrum and the technical support provided by Barcelona Supercomputing Center (FI-2016-3-0006 "New frontiers in numerical general relativity"). P. F. and S. T. are supported by the European Research Council Grant No. ERC-2014StG 639022-NewNGR. P. F. is also supported by a Royal Society University Research Fellowship (Grant No. UF140319). M. K. is supported by an STFC studentship. L. L. is supported by NSERC, CIFAR and Perimeter Institute. This work has received funding from the European Union's Horizon 2020 research and innovation programme under the Marie Skłodowska-Curie Grant agreement No. 690904. P.F. and M. K. would like to thank Perimeter Institute for their hospitality during the final stages of this work. Research at Perimeter Institute is supported by the Government of Canada through the Department of Innovation, Science and Economic Development Canada, and by the Province of Ontario through the Ministry of Research and Innovation.

*p.figueras@qmul.ac.uk

†m.kunesch@damtp.cam.ac.uk

"1lehner@perimeterinstitute.ca

${ }^{\S}$ Present address: DeepMind, 5 New Street Square, London, EC4A 3TW, United Kingdom. stun@google.com

[1] B. P. Abbott et al., Phys. Rev. Lett. 116, 061102 (2016).

[2] B. P. Abbott et al., Phys. Rev. Lett. 116, 241103 (2016).

[3] R. P. Kerr, Phys. Rev. Lett. 11, 237 (1963).
[4] S. Aretakis, Phys. Rev. D 87, 084052 (2013) .

[5] H. Yang, A. Zimmerman, and L. Lehner, Phys. Rev. Lett. 114, 081101 (2015).

[6] S. E. Gralla, A. Zimmerman, and P. Zimmerman, Phys. Rev. D 94, 084017 (2016).

[7] R. Gregory and R. Laflamme, Phys. Rev. Lett. 70, 2837 (1993).

[8] L. Lehner and F. Pretorius, Phys. Rev. Lett. 105, 101102 (2010).

[9] R. Emparan and R. C. Myers, J. High Energy Phys. 09 (2003) 025.

[10] R. Emparan and H. S. Reall, Phys. Rev. Lett. 88, 101101 (2002).

[11] H. Elvang, R. Emparan, and A. Virmani, J. High Energy Phys. 12 (2006) 074.

[12] P. Figueras, K. Murata, and H. S. Reall, Classical Quantum Gravity 28, 225030 (2011).

[13] J. E. Santos and B. Way, Phys. Rev. Lett. 114, 221101 (2015).

[14] K. Tanabe, J. High Energy Phys. 02 (2016) 151.

[15] P. Figueras, M. Kunesch, and S. Tunyasuvunakool, Phys. Rev. Lett. 116, 071102 (2016).

[16] K. Tanabe, arXiv:1605.08116.

[17] O. J. C. Dias, P. Figueras, R. Monteiro, J. E. Santos, and R. Emparan, Phys. Rev. D 80, 111701 (2009).

[18] R. C. Myers and M. J. Perry, Ann. Phys. (N.Y.) 172, 304 (1986).

[19] MP BHs are the higher-dimensional analogues of Kerr BHs.

[20] O. J. C. Dias, J. E. Santos, and B. Way, J. High Energy Phys. 07 (2014) 045.

[21] R. Emparan, P. Figueras, and M. Martinez, J. High Energy Phys. 12 (2014) 072.

[22] F. Pretorius, Classical Quantum Gravity 22, 425 (2005).

[23] M. Shibata and H. Yoshino, Phys. Rev. D 81, 104035 (2010).

[24] W. G. Cook, P. Figueras, M. Kunesch, U. Sperhake, and S. Tunyasuvunakool, Int. J. Mod. Phys. D25, 1641013 (2016).

[25] D. Alic, C. Bona-Casas, C. Bona, L. Rezzolla, and C. Palenzuela, Phys. Rev. D 85, 064040 (2012).

[26] A. Weyhausen, S. Bernuzzi, and D. Hilditch, Phys. Rev. D 85, 024038 (2012).

[27] D. Alic, W. Kastaun, and L. Rezzolla, Phys. Rev. D 88, 064049 (2013).

[28] See Supplemental Material at http://link.aps.org/ supplemental/10.1103/PhysRevLett.118.151103 for a description of our modified Gamma-driver, technical details of our apparent horizon finder, and convergence test results, which includes Refs. [29-31].

[29] M. Alcubierre, B. Brugmann, P. Diener, M. Koppitz, D. Pollney, E. Seidel, and R. Takahashi, Phys. Rev. D 67, 084023 (2003).

[30] M. Alcubierre, Introduction to 3+1 Numerical Relativity (Oxford University Press, Oxford, United Kingdom, 2008).

[31] L. Smarr, Phys. Rev. D 7, 289 (1973).

[32] D. Brown, O. Sarbach, E. Schnetter, M. Tiglio, P. Diener, I. Hawke, and D. Pollney, Phys. Rev. D 76, 081503 (2007).

[33] D. Brown, P. Diener, O. Sarbach, E. Schnetter, and M. Tiglio, Phys. Rev. D 79, 044023 (2009).

[34] J. Thornburg, Living Rev. Relativ. 10, 3 (2007). 
[35] S. Tunyasuvunakool, $\mathrm{PhD}$ Thesis, University of Cambridge, 2016, https://doi.org/10.17863/CAM.7743.

[36] K. Clough, P. Figueras, H. Finkel, M. Kunesch, E. A. Lim, and S. Tunyasuvunakool, Classical Quantum Gravity 32, 245011 (2015).

[37] M. Adams, P. Colella, D. Graves, J. Johnson, N. Keen, T. Ligocki, D. Martin, P. McCorquodale, D. Modiano, P. Schwartz, T. Sternberg, and B. Van Straalen, Lawrence Berkeley National Laboratory Tech. Report No. LBNL6616E (2015).

[38] Videos can be found at http://grchombo.github.io.
[39] J. L. Hovdebo and R. C. Myers, Phys. Rev. D 73, 084013 (2006).

[40] B. Kleihaus, J. Kunz, and E. Radu, Phys. Lett. B 718, 1073 (2013).

[41] L. Lehner and F. Pretorius, arXiv:1106.5184.

[42] H. Elvang, R. Emparan, and P. Figueras, J. High Energy Phys. 05 (2007) 056.

[43] M. Shibata and H. Yoshino, Phys. Rev. D 81, 021501 (2010).

[44] O. J. C. Dias, G. S. Hartnett, and J. E. Santos, Classical Quantum Gravity 31, 245011 (2014). 